\title{
Correction of quasi-static wavefront errors for ELT with two sequential DMs
}

Laurent Pueyo ${ }^{1, a}$, Stuart B Shaklan ${ }^{1}$, Amir Give'on ${ }^{1}$, Mitchell Troy ${ }^{1}$, N. Jeremy Kasdin ${ }^{2}$, Jason Kay ${ }^{2}$, Tyler Groff ${ }^{2}$, Michael McElwain ${ }^{2}$, and Remi Soummer ${ }^{3}$

1 Jet Propulsion Laboratory, California Insitute of Technology, 4800 Oak Grove Drive, Pasadena, CA, 91109, USA

2 Mechanical and Aerospace Engineering, Princeton University, Princeton, NJ, 08544, USA

3 Space telescope Science Institute, 3700 San Martin Drive, Baltimore, MD 21218, USA

\begin{abstract}
Detection and spectral characterization of extrasolar planets with Extremely Large Telescopes (ELT) will require the ability to image very faint objects with a wide spectral bandwidth. Such a goal implies the development of new exquisite wavefront control techniques in order to calibrate and correct both the amplitude and phase components of the residual quasi-static halo that remains after the AO loop. Here we focus on the correction stage at time scales orders of magnitude slower than the $\mathrm{AO}$ refresh rate. We report laboratory results that exhibit four important features of instrument responses necessary for exo-planet characterization: post coronagraphic, high contrast, broadband, symmetric Point Spread Functions. This experiment was conducted in the Princeton University High Contrast Imaging Laboratory, equipped with a shaped pupil coronagraph and two sequential Boston Micromachines Kilo-DMs. In this paper we first review the theory and algorithms underlying one and two DM wavefront controllers and show why a second DM ultimately leads to improved broadband performances. We then present an experimental validation of such a two DM amplitude and phase controller.
\end{abstract}

\section{Introduction}

The recent discovery and imaging of a planetary system orbiting around HR8799 [1] has been an important milestone in the ongoing research effort aimed at increasing the sensitivity of ground based telescopes to faint planetary companions orbiting around nearby stars. Most of the eight meter class observatories are currently developing instruments that couple Extreme Adaptive Optics and coronagraphy to obtain deeper contrast at closer angular separations, for example $10^{-7}$ at 100 mas [2], [3], [4]. The ultimate contrast limit of these instruments will be set by the presence of quasi-static speckles, mainly due to non common path optical imperfections within the AO system [5] [6] [7]. As a consequence the baseline design of this next generation of exo-planet dedicated instruments includes a coherent subtraction of the residual errors, either based on real time measurements [8] or a priori calibration [9]. In the case of Extremely Large Telescopes (ELT), while the large collecting area provides a considerable avenue for sensitivity improvement, the segmentation of the primary mirrors also brings new challenging components to the problem of quasi-static speckles. Indeed, reflectivity non-uniformities from segment to segment create pupil dependent amplitude errors that will be poorly corrected over a wide bandpass if the wavefront controller consists of only one Deformable Mirror (DM) [18].

Compensating for amplitude errors becomes more crucial at high contrast. This problem arises in the case of space based observatories, where the required contrast to detect exo-earths is $10^{-10}$. The inability of a single DM to correct for amplitude aberrations over a broad band is one of the limitation of such space based observatories [10] [11]. In Pueyo et.al [12] we showed how the solution originally proposed by Shaklan et.al [13] could circumvent the problem of amplitude errors. By taking advantage of the phase to amplitude conversion in a sequential DM architecture, one can obtain a controllable wavelength independent lever to correct for amplitude errors. This phase-to-amplitude mixing is also

a e-mail: lpueyo@jpl.nasa.gov

This is an Open Access article distributed under the terms of the Creative Commons Attribution-Noncommercial License, which permits unrestricted use, distribution, and reproduction in any noncommercial medium, provided the original work is properly cited. 
known as the Talbot effect.

In section 2 we present the application of such a sequential DM architecture to compensate for the static telescope errors of an ELT. Here we focus on the example of TMT and show simulations of the expected performances of such a controller used within a very high contrast, narrow field of view exoplanet imaging instrument. In section 3 we present an experimental validation of this architecture. This validation was obtained with a Shaped Pupil Coronagraph [14] in a laboratory dedicated to technology demonstration for space based observatories, but is scalable to ground based architectures.

\section{Performance of a two DM wavefront controller with TMT}

\subsection{Control of amplitude errors with two sequential DMs}

While the physics of phase to amplitude conversion, and thus amplitude correction using two sequential DMs is well known [12] [13], we show here how to integrate it in a wavefront control algorithm that is based on estimations using the detector in the final focal plane. Such an estimate is critical to minimize the impact of non-common path errors. In this section we assume that we can obtain a perfect estimate of the electrical field at the final focal plane using, for instance, DM diversity measurements [15]. We also assume that DM2 is conjugate to the pupil of the telescope, with the apodising mask and the Lyot stop in the case of an APLC, and that DM1 is separated from the pupil plane by a distance $z$. We write the relationship between the pupil plane before the coronagraph and the final image plane using a linear operator $C$. We are looking to cancel the intensity in the final image plane, written as $\left|E_{f}\right|^{2}$, in the locations where a potential planet flux is expected to be detected. Such a problem is infinite-dimensional and made tractable by approximating the DM surface height by a finite sum of basis functions $\psi(x, y)=\lambda_{0} \sum_{k=1}^{N} a_{k} f_{k}(x, y)=X \bullet F(x, y)$, where $\lambda_{0}$ is the central wavelength in the band considered. The problem is now to choose the coefficients $a_{k}$ to achieve the desired performance. The matrix of actuator commands is written as a concatenation of the modal coefficients of each DM, $X=\left[X^{(1)} X^{(2)}\right]$. We we are seeking to solve for the smallest deformation on the DMs that will achieve a target contrast $10^{-C}$, which leads to the following optimization problem:

$$
\begin{aligned}
& \operatorname{minimize} \frac{1}{2} \sum_{k=1}^{N} a_{k}^{2} \\
& \text { subject to } \mathcal{E}_{\mathcal{S}} \leq 10^{-C}
\end{aligned}
$$

Where $\mathcal{E}_{\mathcal{S}}$ is the integrated intensity in region of interest of the final image plane. This corresponds to a "stroke minimization" problem, where we solve for the minimal actuator displacement under a contrast constraint. If we use a first-order Taylor expansion of the effect of the DM around the command $X$, then the intensity in the final image plane at the $\mathrm{n}$ th iteration is a quadratic function of $\delta X_{n+1}$, where $X_{n+1}=X_{n}+\delta X_{n+1}$. We thus write the following quadratic subprogram at the $\mathrm{n}$ th iteration:

$$
\begin{array}{ll}
\text { minimize } & \frac{1}{2}\left(X_{n}+\delta X_{n+1}\right)^{T}\left(X_{n}+\delta X_{n+1}\right) \\
\text { subject to } & \delta X_{n+1}^{T} M_{0} \delta X_{n+1}+B_{n} \delta X_{n+1}+d_{n} \leq 10^{-C}
\end{array}
$$

We do not delve here in the details of such a reduction that are presented in [16]. The main underlying assumptions are approximating $J_{n}$, the vector of partial derivatives of the field at $M 2$ with respect to $X$, by a constant value $J_{0}[16]$, operating around $X=0$, neglecting cross talk between the DMs and the aberration, and using the angular spectrum approximation for the effect of the out of pupil DM. All these approximations slow down the convergence rate of the algorithm but circumvent the high computational cost associated with the evaluation of the sensitivity matrix at each iteration. The coefficients of the quadratic form are

$$
d_{n}=\left\langle E_{f}, E_{f}\right\rangle_{\mathcal{S}}
$$


Laurent Pueyo et al.: Correction of quasi-static wavefront errors for ELT with two sequential DMs

$$
\begin{aligned}
B_{n} & \left.=2 \mathfrak{R}\left[\left\langle E_{f}, C\left\{J_{0}^{(1)}\right\}\right\rangle_{\mathcal{S}}\left\langle E_{f}, C\left\{A J_{0}^{(2)}\right)\right\}\right\rangle_{\mathcal{S}}\right] \\
M_{0} & =\left[\begin{array}{ll}
\left\langle C\left\{J_{0}^{(1)}\right\}, C\left\{J_{0}^{(1)}\right\}\right\rangle_{\mathcal{S}} & \left\langle C\left\{J_{0}^{(1)}\right\}, C\left\{J_{0}^{(2)}\right\}\right\rangle_{\mathcal{S}} \\
\left\langle C\left\{J_{0}^{(2)}\right\}, C\left\{J_{0}^{(1)}\right\}\right\rangle_{\mathcal{S}}\left\langle C\left\{J_{0}^{(2)}\right\}, C\left\{J_{0}^{(2)}\right\}\right\rangle_{\mathcal{S}}
\end{array}\right]
\end{aligned}
$$

When the angular spectrum is small, $\frac{\pi \lambda z}{D^{2}}\left(\xi^{2}+\eta^{2}\right) \ll 1$, where $(\xi, \eta)$ is the units system in the image plane, and if we rearrange the DM commands as $\tilde{X}=\left[\begin{array}{ll}\tilde{X}_{1} & \tilde{X}_{2}\end{array}\right]=\left[\begin{array}{ll}X_{1} & X_{1}+X_{2}\end{array}\right]$, the sensitivity matrix $M$ becomes

$$
C\left\{\tilde{J}_{0}\right\} \cong\left[-\frac{2 \pi^{2} \lambda_{0} z}{D^{2}}\left(\xi^{2}+\eta^{2}\right) C\left\{F^{(1)}\right\} i \frac{2 \pi \lambda_{0}}{\lambda}\left(C\left\{F^{(1}\right\}+C\left\{F^{(2)}\right\}\right)\right]=\left[C\left\{J^{(1)}{ }_{0}\right\} C\left\{\tilde{J^{(2)}}{ }_{0}\right\}\right]
$$

Because $F^{(j)}(x, y)$ and $A(x, y)$ are real functions in the pupil plane, $C\left\{F^{(1}\right\}$ and $C\left\{F^{(2}\right\}$ feature a Her-
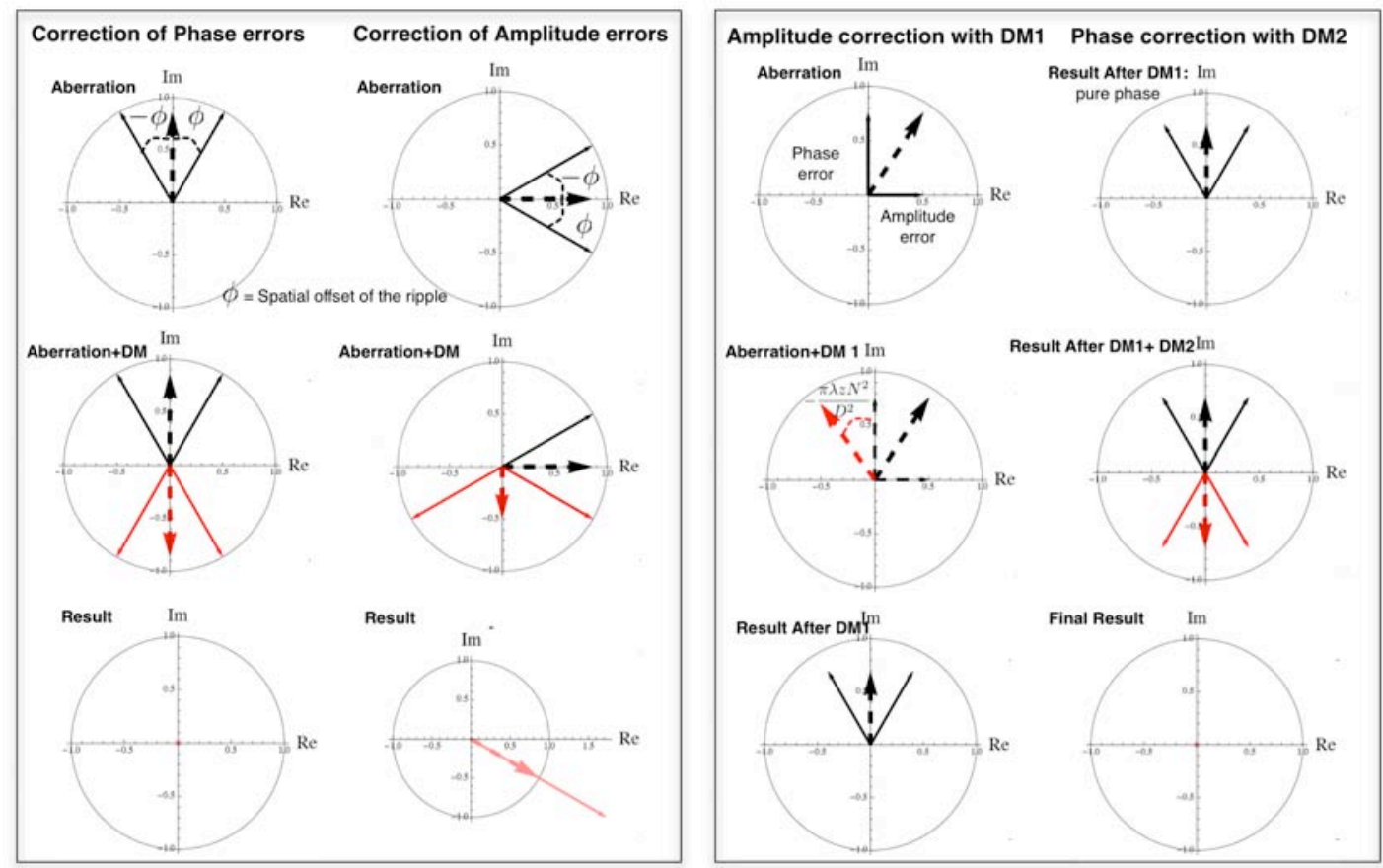

Fig. 1. Left: Phasors representation of a half dark hole generation using a single DM. Right: Phasors representation of a symmetric dark hole generation using two sequential DM

mitian symmetry in the image plane. Thus $C\left\{J^{(1)} 0\right\}$ provides a lever that corrects for Hermitian field distributions in the image plane while $C\left\{\tilde{J}^{(2)} 0\right\}$ provides a lever that corrects for anti-Hermitian distributions. It is the combination of these two independent Hermitian and anti-Hermitian levers that allows us to create symmetric monochromatic dark holes, as shown on Fig. 1. Moreover each of these two independent levers has a different chromatic behavior: the Hermitian one scales as $\lambda^{0}$ and the anti-Hermitian one scales at $1 / \lambda$. In terms of wavefront errors this means that a two sequential DM controller can correct over a broadband for what are commonly called amplitude and phase errors in the pupil plane.

\subsection{Coronagraph design}

We now delve into the details of our simulations that illustrate how well a two sequential DM wavefront controller can correct for reflectivity non uniformities in an ELT. Here we use the example of 
the TMT geometry. In order to obtain contrasts below $10^{-8}$ within 60 mas of the host star, thus enabling detection of close-in faint detection of exo-planets, we first need to eliminate the static impulse response of the telescope finite aperture using a coronagraph. In this paper we choose an APLC, since the apodising screen of such an apparatus can be design to mitigate the secondary obstruction and its support structures [17]. For a given focal plane stop size, here a diameter of $10 \lambda / D$ at the largest wavelength in the bandpass, the apodising mask was calculated using an iterative procedure that seeks the eigen-function of the coronagraph operator that has the largest eigenvalue [17]. Note that one could also embed this iterative algorithm in a global optimization that also finds the best focal plane stop diameter for a given pupil geometry and bandpass, and thus yielding a throughput higher than the 10 percent shown here. Fig. 2 shows the design resulting form this iterative procedure, which features a 55 mas angular resolution at the largest wavelength and an overall contrast of $10^{-8}$.
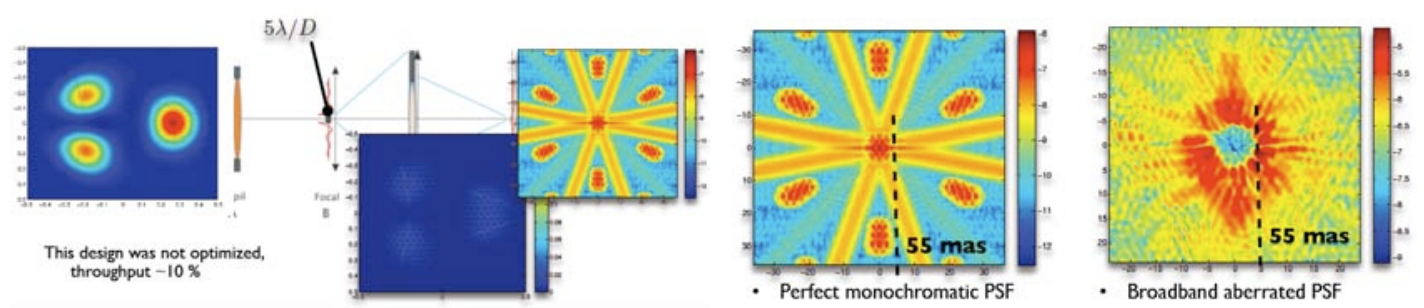

Fig. 2. Left panel, APLC coronagraph applied to a TMT pupil. Left: apodising mask obtained using and iterative algorithm applied to the telescope pupil, note that the apodiser is build around the secondary structures. The image plane mask size was chosen to be $5 \lambda_{0} / D$ radius. Middle: Lyot plane at the wavelength $\lambda_{0}$ for this design, note that this field distribution is exactly proportional to the original apodising mask. Right: PSF obtained in the absence of aberrations. Right panel, Left: perfect monochomatic PSF at the design wavelength. Right: Aberrated PSF

\subsection{Sequential DMs to correct amplitude errors}

In the numerical work presented here we inject the pupil of the coronagraph with a static wavefront that is composed of phase and amplitude errors due to telescope imperfections. In reality they will not be completely static and will be superposed to the ExAO phase residuals, thus requiring a wavefront estimate obtained at faster time scales than the science exposure. A post-coronagraphic calibration device [8], that does not introduce non common path errors since the bulk of the starlight has been cancelled by the coronagraph, could provide such wavefront estimates: in this case the simulations presented here can be seen as the result of a two sequential DM amplitude and phase actuator that is controlled using estimates from such a calibration device. The phase errors used in these simulations were $200 \mathrm{~nm} \mathrm{rms}$ with a drop off of $f^{-3 / 2}$. The amplitude errors were chosen to represent a 1 percent reflectivity errors from segment to segment. We work in the $\mathrm{H}$ band, $\lambda=1.6 \mu \mathrm{m} \pm 0.16 \mu \mathrm{m}$. While the aberrated contrast is above $10^{-6}$, Fig. 3 shows that the controller can retrieve the original coronagraphic floor of $10^{-8}$ over the full 20 percent bandwidth. Using two Deformable Mirrors in series is a promising solution to the problem of reflectivity non-uniformities in ELT and thus enabling ground-based observations of faint exo-planets close to their parent star. In the next section we present an experimental validation of such a concept.

\section{Experimental results}

The optical layout of the Princeton High Contrast Imaging Laboratory is shown in Fig. 4. It consists of two six-inch off-axis parabolic mirrors to collimate and refocus the beam, a shaped pupil coronagraph, and two sequential DMs for control. The DM(s) are $1 \mathrm{~cm}$ on a side, and neither is located in a plane conjugate to the pupil. For the experiment presented here, we use the image plane camera and and 


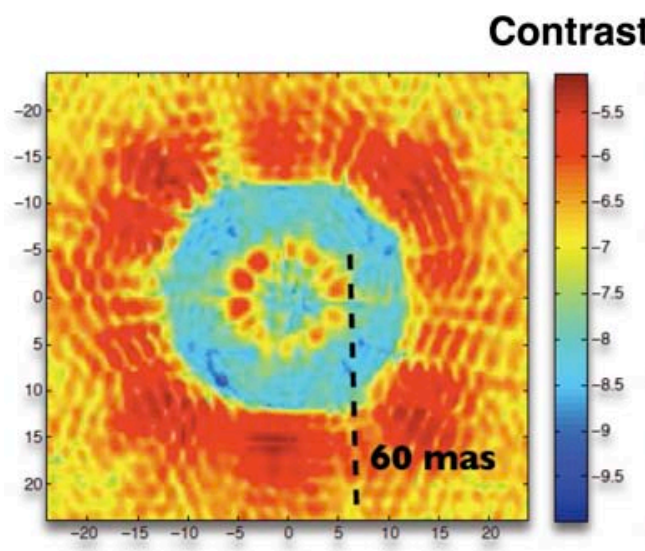

Angular separation in units of $\lambda_{0} / \mathrm{D}$

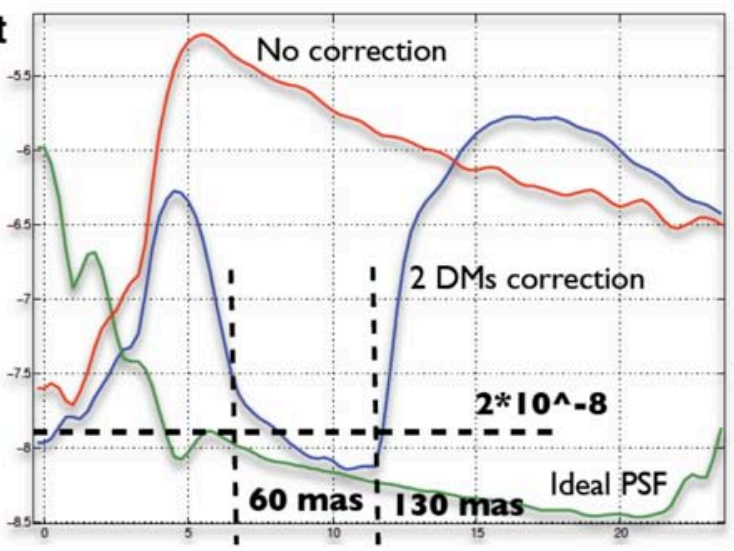

Angular separation in units of $\lambda_{0} / \mathrm{D}$

Fig. 3. Left: Corrected PSF. Right: Radial average
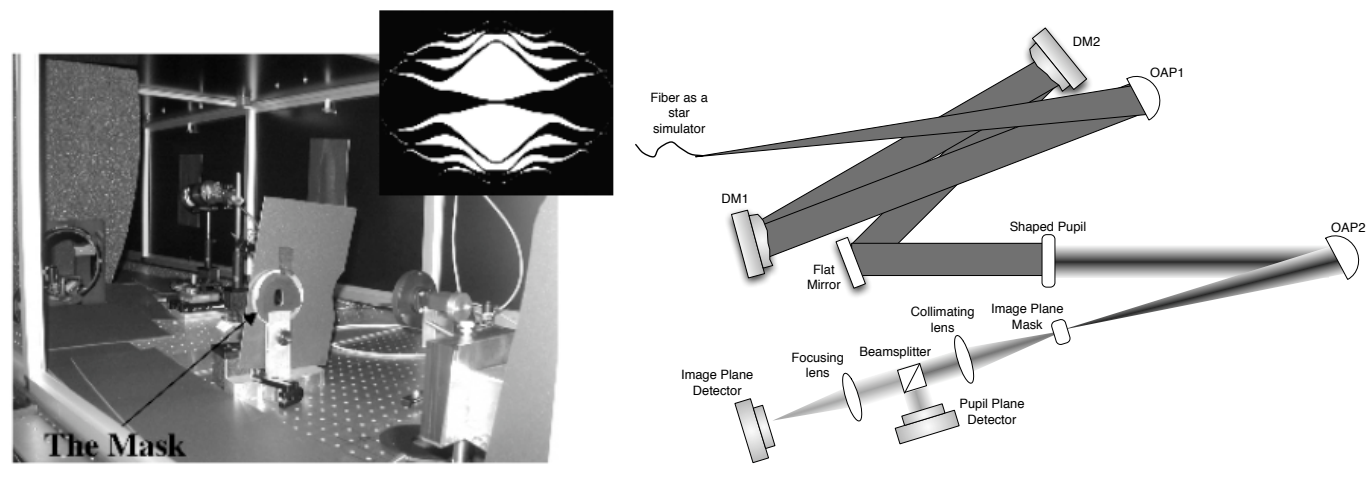

Fig. 4. Left: Picture of the laboratory setup. Right: Layout of the laboratory setup

only use diversity on DM1 to estimate the aberrated field. In this section, we present the first results of a symmetric dark hole using two DMs in sequence to correct for errors on both sides of the image plane. This experiment was performed in monochromatic $632.8 \mathrm{~nm}$ light using the stroke minimization algorithm as described above. Figure 5 shows the aberrated image prior to correction as well as the image after 60 iterations of the stroke minimization correction algorithm. In addition, the figure shows a contrast plot as a function of iteration. The Dark Hole was specified in the wavefront control algorithm to be from 7-10 $\lambda / D$ in the $x$-direction and -2.5-2.5 $\lambda / D$ in the $y$-direction. The average contrast between the two sides of the image plane before any correction is at $1.2 \times 10^{-4}$ with the right side starting out worse than the left side. After 60 iterations, the contrast on both sides of the image has improved to $2.5 \times 10^{-6}$. This figure shows that the stroke minimization algorithm allowed us to improve the on-axis coherent light extinction by almost two orders of magnitude.

\section{Conclusion}

Compensating for reflectivity non-uniformities from segment to segment presents a challenging problem when seeking to image exo-planet using an Extremely Large Telescope. In this paper we developed a wavefront control architecture that, when associated with a well suited coronagraph, enable us 

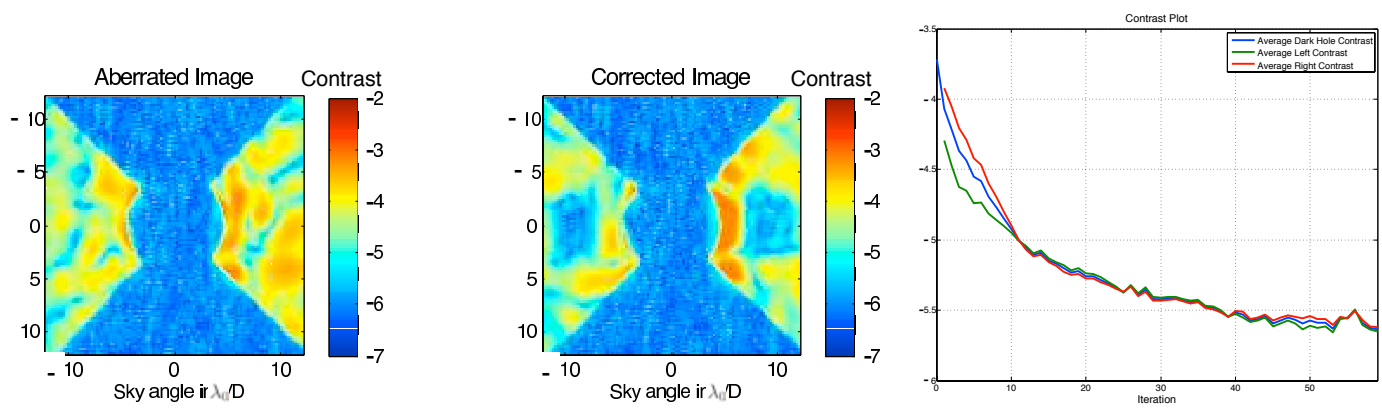

Fig. 5. Left panel: Aberrated image (left) and corrected image (right) of the $2 \mathrm{DM}$ stroke minimization symmetric dark hole experiment. Right panel: A plot of contrast vs. iteration in each of the two dark holes and in the combination of the two.

to reach the required contrast for exo-planet science, even in the presence of amplitude aberrations. We then presented the experimental validation of such an architecture. The current limitation of this experiment is the poor knowledge of the DM surface response which leads to a biased field wavefront estimate when a DM diversity scheme is used. This limitation is scheduled to be addressed in the near future.

\section{Acknowledgements}

The research described in this publication was carried out at the Jet Propulsion Laboratory, California Institute of Technology, under a contract with the National Aeronautics and Space Administration. The first author was supported by an appointment to the NASA Postdoctoral Program at the JPL, Caltech, administered by Oak Ridge Associated Universities through a contract with NASA. Part of this material is also based upon work supported by the National Science Foundation under Award No. AST-0901967.

\section{References}

1. C. Marois et. al, Science 322, pp. 1348-, Nov. 2008.

2. K. Dohlen et.al, SPIE Procs 6269, July 2006.

3. J. R. Graham et.al, ArXiv e-prints 704, Apr. 2007.

4. K. W. Hodapp et.al, SPIE Procs 7014, Aug. 2008.

5. M. P. Fitzgerald and J. R. Graham, ApJ 637, pp. 541-547, Jan. 2006.

6. S. Hinkley et.al, ApJ 654, pp. 633-640, Jan. 2007.

7. R. Soummer and A. Ferrari, ApJl 663, pp. L49-L52, July 2007.

8. J. K. Wallace et.al, SPIE Procs 7015, July 2008.

9. J.-F. Sauvage et.al, JOSA 24, pp. 2334-2346, Aug. 2007.

10. R. A. Brown and C. J. Burrows, Icarus 87, pp. 484-497, 1990.

11. C.Noecker et.al, Procs SPIE 4860, pp. 72-83, 2003.

12. L. Pueyo and N. J. Kasdin, ApJ 666, pp. 609-625, Sept. 2007.

13. S. B. Shaklan and J. J. Green, Applied Optics 45, pp. 5143-5153, July 2006.

14. N. J. Kasdin et.al, Applied Optics 44, pp. 1117-1128, Mar. 2005.

15. A. Give'on, et.al $A A S$ 211, p. 135.20, Dec. 2007.

16. L. Pueyo et.al, Applied Optics, Vol. 48, No. 33, Nov 2009

17. R. Soummer, ApJl 618, pp. L161-L164, Jan. 2005.

18. M. Troy et al. SPIE Procs 6272, July 2006. 